\title{
SOLANUM BETACEUM IMPROVES COGNITIVE FUNCTION BY DECREASING N-METHYL-D-ASPARTATE ON ALZHEIMER RATS MODEL
}

\author{
INDRI SAFITRI ${ }^{1}$, HANIK BADRIYAH HIDAYATI ${ }^{2 *}$, AGUS TURCHAN ${ }^{3}$, SUHARTATI ${ }^{4}$, SITI KHAERUNNISA ${ }^{1}$
}

${ }^{1}$ Department of Biochemistry, Faculty of Medicine, Airlangga University, Indonesia. ${ }^{2}$ Department of Neurology, Airlangga University, Dr. Soetomo General Hospital, Indonesia. ${ }^{3}$ Department of Neurosurgeon, Airlangga University, Dr. Soetomo General Hospital, Indonesia. ${ }^{4}$ Department of Medical Biochemistry, Wijaya Kusuma University, Indonesia. Email: hanikhidayati@yahoo.com

Received: 19 December 2018, Revised and Accepted: 22 July 2019

ABSTRACT

Objective: The purpose of this study was to evaluate the effect of Solanum betaceum towards cognitive function, i.e. memory, and the level of N-MethylD-Aspartate receptor (NMDAR) and brain derived neurothropic factor (BDNF) as a drug candidate therapy for Alzheimer rats model.

Methods: Fifty adult male albino rats were divided into five groups (K0, K1, P1, P2 and P3). Four groups (K1, P1, P2 and P3) of Alzheimer's disease (AD) rats were induced by aluminum chloride with dose $2 \mathrm{~g} / \mathrm{L}$ for 21 days period and three groups (P1, P2 and P3) in 22 $2^{\text {th }}$ day administered parallelly with $100 \mathrm{mg} / \mathrm{kg}$ b.w/day; $200 \mathrm{mg} / \mathrm{kg}$ b.w/day; and $400 \mathrm{mg} / \mathrm{kg}$ b.w/day of $S$. betaceum respectively for 14 days. The level of NMDAR and BDNF was measured by enzyme-linked immunosorbent assay methods, whereas memory was measured by the Morris water maze test.

Results: S. betaceum administration increased cognitive function significantly $(\mathrm{p}=0.037)$ of AD induced-rats by decreasing the time to reach the target of Morris water maze and maintaining the low levels of NMDAR significantly $(\mathrm{p}=0.006)$, but the level of BDNF did not increase significantly ( $\mathrm{p}=0.346)$. These results indicated that ethanol extracts of $S$. betaceum could decrease brain NMDAR and increase cognitive function by promote better memory function but did not significant increased the level of BDNF in AD-induced rats.

Conclusion: This study revealed that the treatment of AD-induced rats with S. betaceum extracts significantly improve memory function and decrease the level of NMDAR.

Keywords: Solanum betaceum, Memory, N-Methyl-D-Aspartate receptor, Brain-derived neurotrophic factor, Aluminum chloride, Alzheimer.

(c) 2019 The Authors. Published by Innovare Academic Sciences Pvt Ltd. This is an open access article under the CC BY license (http://creativecommons. org/licenses/by/4. 0/) DOI: http://dx.doi.org/10.22159/ijap.2019.v11s5.T1015

\section{INTRODUCTION}

Alzheimer's disease (AD), a progressive, irreversible age-related neurodegenerative disease, is characterized by gradually progressive debilitating cognitive decline such as memory loss, disorientation in time and space, difficulty in problems solving, language impairment, psychosocial impairment, behavioral symptoms (hallucinations, paranoia, and delusions), and among others [1-5]. AD is the sixth leading cause of death and a leading cause of dementia among elderly populations of Americans [5]. AD remains a big problem with a significant social, health, and financial burden on society [2]. The latest report infer that $\mathrm{AD}$ affects approximately 5 million Americans and 48 million people worldwide (World Health Organization, 2015) and the incidence of AD continuously and rapidly increase with a new diagnosis being made every $68 \mathrm{~s}[2,6-8]$. The population of the world is rapidly aging, so the number of people with dementia is supposed to increase. $\mathrm{AD}$ is the most common cause of dementia $[2,9]$.

$\mathrm{AD}$ is known as a result from over-production and impaired clearance of $\beta$-amyloid [1]. The extracellular amyloid-beta $(A \beta)$ plaques, intracellular neurofibrillary tangles, large scale of neuronal death and neural atrophy, the loss of synapses, changes in neurotransmitter expression, and reduced neutrophil numbers, which all contribute to cognitive decline in a progressive manner, are the main hallmarks of $\mathrm{AD}[3,10]$. Impairment of memory and other cognitive functions in the initial stages of $\mathrm{AD}$ are associated with changes in the entorhinal cortex and the hippocampus. Around $80 \%$ of the hippocampal neurons may die over the development of the disease, and this progressive loss is manifest in the cognitive impairment and other symptoms seen in patients with $\mathrm{AD}$ [3]. Hippocampus is a critical area of neuronaldamaged in $\mathrm{AD}[7]$.
Hippocampus, a fundamental role of learning and memory, represents the primary region of adulthood neurogenesis and exerts the largest potential for brain neuroplasticity [11]. Brain-derived neurotrophic factor (BDNF) is one of the fundamental neurotrophic factor in learning and memory, particularly expressed ubiquitous in the brain and playing a key regulator role of development, cognition and plasticity of the hippocampus [9,11-15]. BDNF, a synaptic plasticity marker, is important for long-term potentiation (LTP) like mediating the regulation of excitatory synapses during early LTP $[13,16]$. BDNF is secreted at pre- or postsynaptic areas. Synaptic BDNF is secreted in response to activity and can activate pre- and/or post-synaptic TrkB receptors. BDNF increases the exocytosis of glutamate-containing synaptic vesicles presynaptically, whereas postsynaptically BDNFTrkB signaling induces N-Methyl-D-aspartate receptor (NMDAR) phosphorylation [13]. BDNF and NMDA play key role of synaptic plasticity in the hippocampus [13].

Until now pharmacologic management trying to treat $\mathrm{AD}$ is only partial inhibitors rather than curative [10]. AD still a big health problem due to lack of efficacy and adequacy of current treatments [5]. Fundamental strategy to combat $\mathrm{AD}$ is understanding about the underlying neurobiological processes of cognitive decline [17]. Nutrition may be taken into account play an important role to postpone or prevent the development of the disease. There is strong evidence supporting the importance of nutrition in the prevention and management of AD [10]. A classic feature of $\mathrm{AD}$ is dysfunction of hippocampal synaptic plasticity thus modification of synaptic plasticity would be beneficial for memory improvement in AD [7]. Solanum betaceum is promising sources of antioxidants due to their high anthocyanins (ACN) content and associated with beneficial effects of health due to anti-inflammatory effects and improved cognitive behavior [18]. Therefore, the current 
study aimed to determine whether cognitive impairment of AD, neuroplasticity in the hippocampus can be prevented or improved by $S$. betaceum by checking the BDNF and NMDA levels.

\section{METHODS}

\section{Setting}

This study has been conducted in the Animal Research Laboratory of Medical Biochemistry Department Universitas Airlangga, Surabaya city, East Java Province, Indonesia.

\section{Chemicals and reagents}

The level of NMDA and BDNF was obtained using mybiosource. com, California, Sandiego USA, with Cat No.MBS269995 and Cat No. MBS261374, respectively. Aluminum chloride $\left(\mathrm{ACl}_{3}\right)$ has used Cat No.8.01081.0500, MERCK, USA. All of other chemicals and reagents were used of reagent grade and highest purity. Fresh red $S$. betaceum was purchased from local Orchard in Wonosobo, Central Java, Indonesia, identified by Indonesian Institute of Science and processed immediately on arriving at the laboratory.

\section{Animals}

Fifty male, 3 months albino rats with an average weight of $\pm 150-180$ g were used for this study. The animals were acclimatized to the laboratory conditions for 7 days, allowed free access to food and water ad libitum, and maintained under $12 \mathrm{~h}$ light and dark cycles at room temperature.

\section{Grouping and experimental design}

The animals were randomly divided into five groups $(\mathrm{n}=10)$ as follows:

- Group $\mathrm{K}_{0}$ : Negative control

- Group $\mathrm{K}_{1}$ : Aluminum chloride $\left(\mathrm{AlCl}_{3}\right)$

- Group $\mathrm{P}_{1}$ : $\mathrm{AlCl}_{3}$ and ethanol extract of $S$. betaceum $100 \mathrm{mg} / \mathrm{BW}$

- Group $\mathrm{P}_{2}: \mathrm{AlCl}_{3}$ and ethanol extract of $S$. betaceum $200 \mathrm{mg} / \mathrm{kgBW}$

- Group $\mathrm{P}_{3}: \mathrm{AlCl}_{3}$ and ethanol extract of $S$. betaceum $400 \mathrm{mg} / \mathrm{kgBW}$.

$3 \mathrm{~g} / \mathrm{L}$ ( $3 \mathrm{mg} / \mathrm{mL}$ ) of the stock solution of $\mathrm{AlCl}_{3}$ was used in this study. Duration of $\mathrm{AlCl}_{3}$ administration was 21 days. $1^{\text {st }}$ until $21^{\text {th }}$ days, the rats were given $\mathrm{AlCl}_{3}$ and followed by administration of $S$. betaceum in the $22^{\text {nd }}$ until $36^{\text {th }}$ days. This study was carried out in accordance with the guidelines Ethical Clearance provided by the Animal Care and Use Committee, Faculty of Medicine, Universitas Airlangga with certificate No.259/EC/KEPK/FKUA/2018.

\section{Preparation of $S$. betaceum ethanol extract}

Fresh red S. betaceum (Tamarillo) fruits were dried by fresh dryer. Dry powder was extracted by maceration using ethanol solvent for $3 \mathrm{~h} \times 24 \mathrm{~h} 3$ times at room temperature, then separated between filtrate and residue. The ethanol extract was filtered and evaporated with a rotary vacuum evaporator to obtain a viscous extract. The ethanol extract of $S$. betaceum was added to the treatment diet in as a suspension form with aquadest. During the experiment, the extract of $S$. betaceum was simultaneously administered into the treatment group before $\mathrm{AlCl}_{3}$ exposure.

\section{Consolidation training and memory test (Morris water maze)}

The Morris water maze, a water-filled of the round pool, is a well-established apparatus to evaluate hippocampal-dependent spatial learning and memory in rats $[17,19]$. Rats were trained with extramaze visual cues (a colorful poster, a traffic cone, and two black-andwhite construction paper designs) which are placed around the pool. An escape platform was hidden $1 \mathrm{~cm}$ just beneath the surface of the water (Morris, 1984). Most protocols used four start locations: North, South, East, and West. Animals were given a series of daily trials using a random or semi-random set of start locations. Semi-random start position sets were most common, such the four positions were used, with the restriction that one trial each day was from each of the four positions. The rats were gently lowered that the tail-first into the pool with maximum swim time was set to $60 \mathrm{~s}$. If the mouse located the platform before $60 \mathrm{~s}$ had passed, it was immediately removed from the pool, whereas if more than $60 \mathrm{~s}$ of swimming, the mouse was gently guided to the platform and allowed to re-orient to the distal visual cues with $20 \mathrm{~s}$ additional time before being removed from the pool. The memory test was performed with no symbol marks used. The frequency of reaching targets and settling time in the area was recorded and calculated [19]. The value of memory test, as the time required for the rat to reach the target, was expressed in second. The less time to reach the target is indicated good memory of the rats.

\section{Determining level of NMDA and BDNF}

Samples were collected from the left or right hippocampus and analyzed using the enzyme-linked immunosorbent assay (ELISA) method. The fixed hippocampus was homogenized using a sonicator added by buffer lysis and then centrifuged at $15,000 \mathrm{rpm}$ for $15 \mathrm{~min}$ at $4^{\circ} \mathrm{C}$. The supernatant was used to be analyzed. Samples were immediately stored at $-20^{\circ} \mathrm{C}$. The level of NMDA and BDNF in the hippocampus was determined using the ELISA test.

\section{Statistical analysis}

The statistical analysis was performed by one-way ANOVA and KruskalWallis test. The statistical significance between the experimental groups was assessed by least significant difference and Mann-Whitney U-test with $\mathrm{p}<0.05$. Data analysis was used by SPSS ver.23.

\section{RESULTS AND DISCUSSION}

Table 1 summarized about mean, standard deviation, ANOVA test of NMDAR and BDNF of control and treatment group. ANOVA test revealed NMDAR levels between group differs significantly with $\mathrm{p}=0.006$

Table 1: Mean, standard deviation, ANOVA test of NMDAR, BDNF of rats control and experiment group

\begin{tabular}{|c|c|c|c|c|c|c|c|}
\hline \multirow[t]{2}{*}{ Variable } & \multicolumn{5}{|l|}{ Group ${ }^{\#}$} & \multirow[t]{2}{*}{ SI } & \multirow[t]{2}{*}{ ANOVA } \\
\hline & $\mathbf{K}_{0}$ & $\mathrm{~K}_{1}$ & $P_{1}$ & $\mathbf{P}_{2}$ & $\mathbf{P}_{3}$ & & \\
\hline NMDAR & $3.74 \pm 1.12^{\mathrm{a}}$ & $5.73 \pm 0.6^{\mathrm{b}}$ & $3.91 \pm 0.19^{\mathrm{a}}$ & $4.27 \pm 0.56^{\mathrm{a}}$ & $4.30 \pm 0.98^{\mathrm{a}}$ & $\mathrm{ng} / \mathrm{ml}$ & $0.006^{*}$ \\
\hline BDNF & $56.18 \pm 11.122^{a}$ & $36.04 \pm 6.83^{b}$ & $53.51 \pm 2.41^{\mathrm{a}, \mathrm{b}}$ & $50.54 \pm 6.57^{\mathrm{a}, \mathrm{b}}$ & $49.24 \pm 11.82^{\mathrm{a}, \mathrm{b}}$ & $\mathrm{ng} / \mathrm{ml}$ & 0.280 \\
\hline
\end{tabular}

${ }^{*}$ Significantly with $\mathrm{p}<0.05,{ }^{*}$ mean \pm SD, a, different superscript means significant between groups. NMDAR: N-Methyl-D-Aspartate receptor, BDNF: Brain-derived neurotropic factor, SD: Standard deviation

Table 2: Mean, median, minimum, maximum, and of memory test by Morris water maze test of rats control and treatment group

\begin{tabular}{lllllllll}
\hline Variable & Category & \multicolumn{2}{l}{ Group } & \multicolumn{1}{c}{} & \multirow{2}{*}{ SI } & \multirow{2}{*}{ Kruskal-Wallis } \\
\cline { 3 - 7 } & & $\mathbf{K}_{\mathbf{0}}$ & $\mathbf{K}_{\mathbf{1}}$ & $\mathbf{P}_{\mathbf{1}}$ & $\mathbf{P}_{\mathbf{2}}$ & $\mathbf{P}_{\mathbf{3}}$ & & \\
\hline Memory/escape latency & Mean & $10.71^{\mathrm{a}}$ & $35.71^{\mathrm{b}}$ & $15.85^{\mathrm{a}}$ & $15.57^{\mathrm{a}}$ & $16.71^{\mathrm{a}}$ & Second & $0.038^{*}$ \\
& Median & 11 & 27 & 13 & 8 & 16 & & \\
& Minimum & 3 & 20 & 4 & 3 & 3 & & \\
& Maximum & 21 & 83 & 50 & 33 & 51 & & \\
\hline
\end{tabular}

*Significantly with $\mathrm{p}<0.05$, a,b different superscript means significant between groups 
$(\mathrm{p}<0.05)$. BDNF count between group did not differs significantly with $\mathrm{p}=0.280(\mathrm{p}>0.05)$.

Table 2 summarized mean, median, minimum, maximum and of memory test of rats control and treatment group. The distribution of memory test from $\mathrm{K}_{0}$ to $\mathrm{P}_{3}$ is abnormal, thus used Kruskal-Wallis statistic and revealed differs significantly with $\mathrm{p}=0.038(\mathrm{p}<0.05)$. S. betaceum in a dose of $100 \mathrm{mg} / \mathrm{kg}$ b.w. was the best dose rather than $200 \mathrm{mg} / \mathrm{kg}$ b.w. or $400 \mathrm{mg} / \mathrm{kg}$ b.w. for improving memory impairment in AD induced-rats as shown by Morris water maze test.

Administration of $S$. betaceum for group $\mathrm{P}_{1}, \mathrm{P}_{2}$, and $\mathrm{P}_{3}$ rats showed a significant increase with $\mathrm{p}=0.006(\mathrm{p}<0.05)$ in their level of NMDAR (Table 1) and decrease time in memory or escape latency test compared to Group $\mathrm{K}_{1}$ rats which shows the protective effect of memory loss by $S$. betaceum.

It was well established that $\mathrm{AD}$ has been related to debilitating cognitive decline and the most common form of dementia with symptoms such as memory loss, disorientation in time, and space, difficulty solving problems, and among others [5,4]. In this study, we have chosen the fresh $S$. betaceum fruits to evaluate its ameliorative effect against AD. This study was aimed to determine the beneficial effect of $S$. betaceum administration on cognitive function, i.e. memory of AD-rats by determining the value of NMDAR and BDNF levels of the hippocampal brain. Male, 3 months albino rats were used as an animal model in this study due to its translationally appropriate and reproducible model to investigate age-related changes to neural systems and cognition [17].

S. betaceum (Tamarillo) fruits, previously known as Cyphomandra betaceae, are native and exotic fruits of subtropical and high tropical areas like Indonesia. The commonly used of other names are "tomate de palo," tree tomato, and "tomate de árbol." This fruits belongs to the Solanaceae family and Solanum genus (Bohs, 1995) [18,20-22]. S. betaceum has known as ovoid- or egg-shaped fruits with a glossy, thin and various color of the skin such as reddish-brown, purple-red, golden yellow, and orangered to cream-yellow. S. betaceum has orange pulp, dark red seed with sour and bland taste. The pigment of $S$. betaceum has been published, such as carotenoids and ACN $[20,23,24]$. S. betaceum has divided into two until four types which are distinguished according to their skin colour: Purplered (often divided into purple and red) and yellow (often divided into amber and gold). The main difference of $S$. betaceum between the yellow cultivars and purple-red cultivars can be seen in the anthocyanin content, which is greatly higher in the purple-red cultivars [22]. Our study has used red S. betaceum to be given into AD-rat models.

Red S. betaceum is containing rich anthocyanin [22]. Recently, ACN have shown its beneficial effect in memory function. Dietary anthocyanin may positively impact cognitive function and may exert beneficial effect for the prevention and treatment of dementia. Study before, which was conducted by Kent et al., 2015, provided evidence that anthocyaninrich cherry juice consumption improves memory and cognition in older adults with mild-to-moderate dementia. This study was a randomized controlled trial, assessed cognitive outcomes in 49 older adults $(70$ years old) with mild-to-moderate dementia after consumption of $200 \mathrm{~mL} /$ day of anthocyanin-cherry juice within 12 weeks [25]. Hippocampaldependent spatial learning and memory can be evaluated using a well-established apparatus called by The Morris water maze. Our observations on Morris water maze test, showed significant differences between control, AD-model and protective group's rats suggest that oral administration of $S$. betaceum might improve memory impairment and behavioral changes in AD-rat models. The results of our study derive strong support from the previous study that consumption of anthocyanin showed significant improvement in memory and cognitive impairment [25].

Neurobiochemical, it has been known that excessive amounts of $A \beta$ peptide in the brain, particularly $A \beta 42$, are responsible as underlying pathology for $\mathrm{AD}$. There are various of potential links between $\mathrm{A} \beta$ with the NMDA and NMDAR such as NMDA may be a down-stream target of
$A \beta$, meaning that $A \beta$ mediates the function of NMDAR, the signal of NMDA may influence the assembly of A $\beta$ plaques, NMDAR may bind $A \beta$ through direct or indirect interactions and may mediate $A \beta$ activity relative to plasticity and/or synaptic transmission. The pathogenesis of $\mathrm{AD}$ is highly linked with alterations in glutamate signaling, and the tissues affected by $\mathrm{AD}$ contain high densities of glutamatergic neurons. Chronic and moderate activation of NMDA receptors result in excitotoxicity and leading to neurodegeneration. This hypothesis of excitotoxicity is supported by clinical evidence indicating that the NMDAR antagonist memantine slows AD progression. Prolonged Ca2+ elevation suppresses synaptic function, leading to subsequent synaptotoxicity and eventually atrophies; these events correlate with the loss of learning and memory functions in AD. Multiple neurotrophic factors have been demonstrated to enhance defense against excitotoxicity [3]. One of the fundamental neurotrophic factors in learning and memory is BDNF [13]. BDNF played a pivotal regulatory role in the regulation of hippocampal structure, development, maintenance, growth, neuronal survival, differentiation, axon growth, formation of neurons, dendritic remodeling, synaptic transmission and modulation, neurotransmitter release, promotion synaptic growth and plasticity of the hippocampus, and cognition [9,11,13-15]. NMDAR and BDNF of hippocampus play a pivotal role of synaptic plasticity in the hippocampus [13]. Rao et al. observed that the expression of BDNF in AD brains is decreased and NMDAR-medicated excitotoxicity play a key role in the development of $\mathrm{AD}$ [16]. The results of our study showed that in memory- impaired AD-rats induced by $\mathrm{AlCl}_{3}$, $\mathrm{S}$. betaceum administration decrease the level of NMDAR significantly with $\mathrm{p}=0.006(\mathrm{p}<0.05)$, but did not change the level of BDNF with $\mathrm{p}=0.280(\mathrm{p}>0.05)$

\section{Limitation}

Limitation of the present study is designed only for behavioral, BDNF, and NMDAR by ELISA aspects involved in the AD. The remaining parameters, including various biochemical changes, morphometric, cholinergic, histological aspects, and gene expression studies, should be performed.

\section{CONCLUSION}

Overall, treatment of cognitive impairments in AD-induced rats with $S$. betaceum extract showed its potential in improving effect in memory function as showed in the decreasing time to reach the target of Morris water maze test and decreasing of NMDAR levels of the hippocampal rats. This study strongly indicated that extracts of $S$. betaceum fruits could be good natural sources to improve memory function significantly by a different mechanism of action, such as decreasing the level of NMDAR, but not by changing the level of BDNF, in AD-induced rats. Therefore, consumption of $S$. betaceum in daily dietary intake is a one-step action toward the prevention of cognitive impairments in $\mathrm{AD}$ management principle. Further studies are needed to search another parameter involved in memory function of AD-induced rat models.

\section{ACKNOWLEDGMENT}

This study was conducted by the support of Research and Innovation Institute, Faculty of Medicine of Airlangga University, Surabaya, East Java, Indonesia. We would like to thank for all research fundings to the Ministry of Research, Technology and Higher Education, the Republic of Indonesia.

\section{CONFLICTS OF INTEREST}

The authors declare that all of the authors have no conflicts of interest.

\section{REFERENCES}

1. Apostolova LG. Alzheimer disease. Continuum (Minneap Minn) 2016;22:419-34

2. Korolev I. Alzheimer's disease: A clinical and basic science review. Med Stud Res J 2014;4:24-33. Available from: http://msrj.chm.msu. edu/fall-2014-24/.

3. Kocahan S, Doğan Z. Mechanisms of Alzheimer's disease pathogenesis and prevention: The brain, neural pathology, N-methyl-D-aspartate receptors, tau protein and other risk factors. Clin Psychopharmacol Neurosci 2017:15:1-8. 
4. Sanabria-Castro A, Alvarado-Echeverría I, Monge-Bonilla C. Molecular pathogenesis of Alzheimer's disease: An update. Ann Neurosci 2017;24:46-54

5. Cooper EL, Ma MJ. Alzheimer disease: Clues from traditional and complementary medicine. J Tradit Complement Med 2017;7:380-5.

6. Lim HS, Kim BY, Kim YJ, Jeong SJ. Phytochemical allylguaiacol exerts a neuroprotective effect on hippocampal cells and ameliorates scopolamine-induced memory impairment in mice. Behav Brain Res 2018;339:261-8.

7. Zhu G, Yang S, Xie Z, Wan X. Synaptic modification by L-theanine, a natural constituent in green tea, rescues the impairment of hippocampal long-term potentiation and memory in $\mathrm{AD}$ mice. Neuropharmacology 2018;138:331-40

8. Ghanemi A. Alzheimer's disease therapies: Selected advances and future perspectives. Alexandria J Med 2015;51:1-3. Available from: http:// www.sciencedirect.com/science/article/pii/S209050681400092X.

9. Yuede CM, Timson BF, Hettinger JC, Yuede KM, Edwards $\mathrm{HM}$, Lawson JE, et al. Interactions between stress and physical activity on Alzheimer's disease pathology. Neurobiol Stress 2018; 8:158-71.

10. Muñoz Fernández SS, Lima Ribeiro SM. Nutrition and Alzheimer disease. Clin Geriatr Med 2018;34:677-97

11. Park HS, Kim CJ, Kwak HB, No MH, Heo JW, Kim TW. Physical exercise prevents cognitive impairment by enhancing hippocampal neuroplasticity and mitochondrial function in doxorubicin-induced chemobrain. Neuropharmacology 2018;133:451-61.

12. Kennedy KM, Reese ED, Horn MM, Sizemore AN, Unni AK, Meerbrey ME, et al. BDNF val66met polymorphism affects aging of multiple types of memory. Brain Res 2015;1612:104-17.

13. Leal G, Bramham CR, Duarte CB. BDNF and hippocampal synaptic plasticity. Vitamins and Hormones. $1^{\text {st }}$ ed. Amsterdam, The Netherlands: Elsevier Inc.; 2017. p. 153-95.

14. Mattson MP, Maudsley S, Martin B. BDNF and 5-HT: A dynamic duo in age-related neuronal plasticity and neurodegenerative disorders. Trends Neurosci 2004;27:589-94

15. Song JH, Yu JT, Tan L. Brain-derived neurotrophic factor in
Alzheimer's disease: Risk, mechanisms, and therapy. Mol Neurobiol $2015 \cdot 52: 1477-93$

16. Kumar K, Kumar A, Keegan RM, Deshmukh R. Recent advances in the neurobiology and neuropharmacology of Alzheimer's disease. Biomed Pharmacother 2018;98:297-307.

17. McQuail JA, Johnson SA, Burke SN, Bizon JL. Rat Models of Cognitive Aging. Conn's Handbook of Models for Human Aging. $2^{\text {nd }}$ ed. London, United Kingdom: Elsevier Inc.; 2018. p. 211-30. Available from: https://linkinghub.elsevier.com/retrieve/pii/B9780128113530000178.

18. Olaya CM, Castaño MP, Garzón GA. Stability of anthocyanins from Rubus glaucus Benth and Solanum betaceum Cav. dark-red strain as affected by temperature, storage time and water activity. Acta Biol Colomb 2009; 14:141-56

19. Barnhart CD, Yang D, Lein PJ. Using the morris water maze to assess spatial learning and memory in weanling mice. PLoS One 2015; 10:e124521

20. García JM, Prieto LJ, Guevara A, Malagon D, Osorio C. Chemical studies of yellow tamarillo (Solanum betaceum cav.) fruit flavor by using a molecular sensory approach. Molecules 2016;21. pii: E1729.

21. Li Y, Zhang JJ, Xu DP, Zhou T, Zhou Y, Li S, et al. Bioactivities and health benefits of wild fruits. Int J Mol Sci 2016;17. pii: E1258.

22. Schotsmans WC. Tamarillo (Solanum betaceum (Cav.)). Postharvest Biology and Technology of Tropical and Subtropical Fruits. Mangosteen to White Sapote. Vol. 4 . Sawston: Woodhead Publishing Limited; 2011. p. 427-42e.

23. Gannasin SP, Adzahan NM, Hamzah MY, Mustafa S, Muhammad K. Physicochemical properties of tamarillo (Solanum betaceum cav.) hydrocolloid fractions. Food Chem 2015;182:292-301.

24. Osorio C, Hurtado N, Dawid C, Hofmann T, Heredia-Mira FJ, Lucía A. Chemical characterisation of anthocyanins in tamarillo (Solanum betaceum Cav.) and Andes berry (Rubus glaucus Benth.) fruits. Food Chem 2012;132:1915-21.

25. Kent K, Charlton K, Roodenrys S, Batterham M, Potter J, Traynor V. Consumption of anthocyanin-rich cherry juice for 12 weeks improves memory and cognition in older adults with mild-to-moderate dementia. Eur J Nutr 2017;56:333-41. 